Review Article

\title{
Atherosclerosis and Atheroma Plaque Rupture: Imaging Modalities in the Visualization of Vasa Vasorum and Atherosclerotic Plaques
}

\author{
Zhonghua Sun \\ Discipline of Medical Imaging, Department of Imaging and Applied Physics, Curtin University, P.O. Box U1987, \\ Perth, WA 6845, Australia \\ Correspondence should be addressed to Zhonghua Sun; z.sun@curtin.edu.au
}

Received 31 August 2013; Accepted 30 December 2013; Published 11 February 2014

Academic Editors: M. D. Cheitlin and P. Quax

Copyright (C) 2014 Zhonghua Sun. This is an open access article distributed under the Creative Commons Attribution License, which permits unrestricted use, distribution, and reproduction in any medium, provided the original work is properly cited.

Invasive angiography has been widely accepted as the gold standard to diagnose cardiovascular pathologies. Despite its superior resolution of demonstrating atherosclerotic plaque in terms of degree of lumen stenosis, the morphological assessment for the plaque is insufficient for the analysis of plaque components, and therefore, unable to predict the risk status or vulnerability of atherosclerotic plaque. There is an increased body of evidence to show that the vasa vasorum play an important role in the initiation, progression, and complications of atherosclerotic plaque leading to major adverse cardiac events. This paper provides an overview of the evidence-based reviews of various imaging modalities with regard to their potential value for comprehensive characterization of the composition, burden, and neovascularization of atherosclerotic plaque.

\section{Introduction}

The development of atherosclerosis is associated with structural and functional changes in the vascular wall [1]. Adventitial vasa vasorum are functional endarteries that are normally present on the adventitial side of the arteries $[2,3]$. The main functions of the vasa vasorum are to deliver nutrients to the vessel wall and to remove waste products or noxious substances [4]. The density of this vascular network increases comparably with the natural growth of the vessel wall and is closely associated with the progression of vessel wall disease, particularly those diseases involving inflammation, including atherosclerosis and diabetes [5].

Adventitial vasa vasorum has been shown to participate in the process of atherogenesis and atherosclerotic plaque progression [6-8]. Extensive research has been conducted on experimental animals and human autopsy data in the past 20 years to support the hypothesis that vasa vasorumderived plaque neovascularization is intimately associated with atherosclerotic plaque progression and destabilization.

Vasa vasorum can be visualized directly or indirectly by several imaging modalities in experimental models and clinical settings, including microcomputed tomography (CT), contrast-enhanced ultrasound (CEUS), intravascular ultrasound (IVUS), optical coherence tomography (OCT), and contrast-enhanced magnetic resonance imaging (CE-MRI). These imaging techniques provide a unique opportunity to demonstrate the normal anatomy of vasa vasorum and monitor progressive pathophysiological developments associated with atherosclerosis due to vasa vasorum injury. This review paper focuses on different imaging modalities for identification and visualization of vasa vasorum and neovascularization, as well as characterization of plaque components.

\section{Imaging Modalities in the Visualization of Vasa Vasorum and Atherosclerotic Plaques}

2.1. Micro-CT. Micro-CT is an ex vivo imaging modality capable of achieving a spatial resolution on the order of $20 \mu \mathrm{m}^{3}$, which enables acquisition of high spatial resolution images of vasa vasorum in human and animal models $[9,10]$. A micro-CT scanner generates 3D images consisting of up to a billion cubic voxels, with each $5-25 \mu \mathrm{m}$ on a side having isotropic spatial resolution. Its main components 
include a spectroscopic X-ray source that produces selectable primary emission peaks at $\sim 9,18$, or $25 \mathrm{keV}$ and a fluorescing thin crystal plate that is imaged with a lens onto a $2.5 \times 2.5$ $\mathrm{cm}, 1024 \times 1024$-pixel charge-coupled device (CCD) detector array [11]. The specimen is positioned close to the crystal and is rotated around $360^{\circ}$ between each X-ray exposure and its CCD recording, with tomographic reconstruction algorithms applied to these recorded images, which are used to generate $3 \mathrm{D}$ images of the specimen. In order to differentiate grayscale values in the media and adventitia area in arteries, the voxel sizes should be smaller than $200 \mu \mathrm{m}^{3}$ to allow quantitative analysis of vasa vasorum in the coronary wall [12]. The latest multislice CT scanners provide voxel sizes greater than $350 \mu \mathrm{m}^{3}$ [13], which make it difficult to visualize the vasa vasorum.

Kwon et al. in their early study showed the 3D anatomy of vasa vasorum in both normal and balloon-injured coronary arteries [14]. In their study, the first-order vasa vasorum arising from the lumen of the coronary artery which ran longitudinally along the media-adventitial layer and the smaller second-order vasa vasorum arising from branches of first-order vasa vasorum were clearly demonstrated in normal pig arteries. Their results showed that the mean diameter of normal first-order vasa vasorum was $160.92 \pm$ $5.10 \mu \mathrm{m}$ and of second-order vasa vasorum, $67.99 \pm 2.72 \mu \mathrm{m}$ $(P<0.0001)$, while in the injured coronary arteries, the mean diameter of first- and second-order vasa vasorum was $141.11 \pm 5.87 \mu \mathrm{m}$ and $101.59 \pm 1.49 \mu \mathrm{m}$, respectively, with significant differences between normal and injured arteries $(P<0.05)$. In addition, marked adventitial neovascularization was observed in the injured porcine coronary arteries when compared to the normal coronary arteries in terms of vessel wall area $\left(4.85 \pm 0.35 \mathrm{~mm}^{2}\right.$ versus $11.24 \pm 0.32 \mathrm{~mm}^{2}$, $P=0.0001$, resp.) and density of vasa vasorum $(3.16 \pm$ 0.17 per $\mathrm{mm}^{2}$ versus $1.90 \pm 0.06$ per $\left.\mathrm{mm}^{2}, P=0.0001\right)$. Later reports using micro-CT further confirmed the clinical value of this high-resolution noninvasive imaging modality in terms of quantitative assessment of vasa vasorum distribution in the coronary arteries and its role in the development of atherosclerosis [15-21].

There is a growing body of evidence showing that vasa vasorum neovascularization plays an important role in the progression and complications of atherosclerosis. In an experimental hypercholesterolemia study, Herrmann et al. used 3D micro-CT to demonstrate an increase in coronary vasa vasorum density within the first 4 weeks prior to the development of endothelial dysfunction, therefore, suggesting a role for vasa vasorum neovascularization in the initiation of atherosclerosis [15]. The density of vasa vasorum was significantly increased in animals on a hypercholesterolemic diet for 2 and 4 weeks $\left(4.88 \pm 2.45\right.$ per $\left.\mathrm{mm}^{2}\right)$ and 6 and 12 weeks $\left(4.50 \pm 1.37\right.$ per $\left.\mathrm{mm}^{2}\right)$ when compared to the control group $\left(2.97 \pm 1.37\right.$ per $\left.\mathrm{mm}^{2}\right)$. This is confirmed by experimental studies using antiangiogenic drug to inhibit inflammatory effects, consequently inhibiting vasa vasorum neovascularization [16-19]. Gössl et al. used micro-CT to compare and analyse vasa vasorum density in normal and high-cholesterol diet animals [17]. They reported that vasa vasorum density and intima-media thickness were significantly increased in the hypercholesterolemic animals when compared to the normal group $\left(6.4 \pm 0.7 \mathrm{~mm}^{2}\right.$ versus $2.7 \pm 0.3 \mathrm{~mm}^{2}, 0.62 \pm 0.09 \mathrm{~mm}$ versus $0.28 \pm 0.02 \mathrm{~mm}$, corresponding to vasa vasorum density and intima-media thickness, resp., $P<0.05)$. Furthermore, prevention of vasa vasorum neovascularization and significant reduction of vascular endothelial growth factor were achieved through administration of anti-angiogenic drug, which led to a significant reduction of vascular area fraction, intima-media thickness, and endothelial exchange surface within the coronary artery wall [4, 17-19]. These observations contribute to the inhibition of neointima proliferation, which in turn inhibits the development of early atherosclerosis [20-22].

In addition to visualization of normal anatomy of vasa vasorum distribution and pathological change along the artery wall, micro-CT is also able to detect segmental vasa vasorum neovascularization in the coronary artery. Based on 20 coronary segments from 15 autopsy patients, Gössl and colleagues in their study found that vasa vasorum density was higher in artery segments with nonstenotic plaques and with noncalcified stenotic plaques than that in the normal segments $\left(3.36 \pm 0.45 \mathrm{~mm}^{2}, 3.73 \pm 1.03 \mathrm{~mm}^{2}\right.$ versus $1.16 \pm$ $0.21 \mathrm{~mm}^{2}, P<0.01$ ), while in the presence of significant calcification, vasa vasorum spatial density decreased significantly to the levels similar to those observed from normal coronary segments $\left(0.95 \pm 0.21 \mathrm{~mm}^{2}\right)$ (Figure 1) [23] Coronary calcification is regarded as a reliable indicator of atherosclerosis and its extent is related to the plaque burden, but not to the degree of obstruction $[24,25]$. However, the mechanism of the calcification process, whether it is part of the inflammatory damage or part of the repair process, remains to be understood [26].

In summary, micro-CT has demonstrated superior spatial resolution which allows for quantitative imaging of the spatial and temporal distribution of vasa vasorum in the aorta and coronary artery $[15,17,23,27]$. Vasa vasorum parameters including vasa vasorum count, vasa vasorum spatial density, vasa vasorum vascular area fraction, and vasa vasorum endothelial surface fraction can be reliably evaluated with use of micro-CT $[18,26,27]$. The increase in arterial wall opacity due to increased vascularity of the vasa vasorum can also be detected on micro-CT images, and this serves as an index of the angiogenesis that takes places in early plaque development [28]. These research findings corroborate the clinical value of micro-CT imaging in the investigation of atherosclerosis due to vasa vasorum injury and its role in the initiation and progression of atherosclerotic plaques.

2.2. Contrast-Enhanced Ultrasound. Recently, ultrasound contrast agents have been developed and used to visualize the carotid artery vasa vasorum and neovascularization of atherosclerotic plaques. Contrast-enhanced ultrasound (CEUS) is a promising noninvasive modality for visualization of plaque neovascularization [29]. Microbubble ultrasound contrast agents are confirmed to be clinically useful in enhancing ultrasound images and improving the diagnostic accuracy [30]. The real-time CEUS imaging enables 


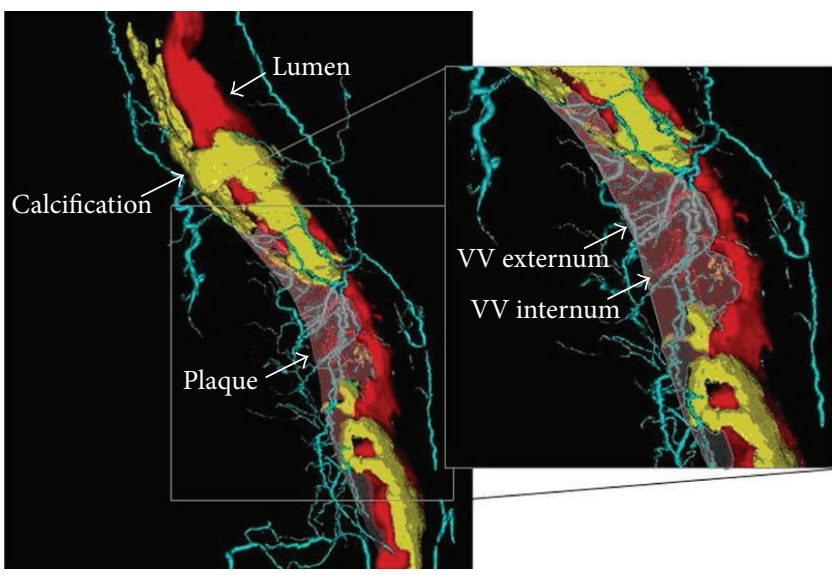

Figure 1: Volume-rendered micro-CT imaging of a coronary plaque and vasa vasorum. Volume-rendered 3D micro-CT image of a right coronary artery showing the main coronary lumen in red; noncalcified and calcified plaque areas are indicated by the arrows (noncalcified plaque is transparent). Vasa vasorum (VV) are shown in light blue (VV externa) or red (VV interna, directly originating from the main lumen). Reprint with permission from [23].

a dynamic evaluation of microvascular assessment of the spatial and temporal heterogeneity of adventitial and intraplaque vasa vasorum [29-32]. The usefulness and reliability of CEUS have been validated by previous studies in animals and humans that showed the degree of plaque neovascularization which is correlated closely with density of neovessels [33-35].

Schinkel et al. demonstrated the feasibility of imaging vasa vasorum using CEUS in their animal experiments. CEUS is able to detect and monitor the progression of intima-media thickness and the density of the vasa vasorum network in the follow-up of swine models of atherosclerosis $(0.22 \pm 0.05 \mathrm{~mm}$ versus $0.45 \pm 0.06 \mathrm{~mm}$ at baseline, $P<0.001)$ (Figure 2) [36]. Clinical reports on patient data further corroborated the feasibility of using CEUS in the visualization of adventitial neovascularization. Giannoni et al. in their pilot study showed that CEUS has potential value in imaging plaque neovascularization (73 plaque specimens) between symptomatic and asymptomatic patients. In patients with acute symptomatic disease, CEUS detected a specific pattern of diffuse contrast enhancement at the base of the carotid plaques, close to the adventitial layer of the artery wall. This corresponded to a high density of small diameter microvessels, indicating the presence of plaque angiogenesis, despite inclusion of only 9 symptomatic patients in their group [37]. Their findings are further validated by Xiong et al.s study (133 plaques) which involved comparing 35 symptomatic patients with 69 asymptomatic patients using CEUS [38]. A higher prevalence of carotid plaque enhancement was observed in symptomatic patients than in the asymptomatic group, showing good correlation between the degree of contrast enhancement and patient symptoms. Their results showed that enhanced intensity in the plaque $(13.9 \pm 6.4 \mathrm{~dB})$ and the ratio of enhanced intensity in the plaque to that in the lumen of the carotid artery $(0.54 \pm 0.23)$ in symptomatic patients were significantly higher than those in asymptomatic patients $(8.8 \pm 5.2 \mathrm{~dB}$ and $0.33 \pm 0.19, P<0.001)$. These findings confirm that CEUS may be used for plaque risk stratification and for the assessment of progression of atherosclerosis [38-40].

Traditional CEUS has limited value in quantitative assessment of progression or regression of vasa vasorum due to the effect of microbubble concentration in the blood pool, which is variable over time and between subjects. Lee et al. in their recent study used maximum intensity projection (MIP) processing of CEUS images to enhance diagnostic sensitivity for detection of microvessels in vitro animal studies [41]. Their MIP technique allows for detection of microvascular linear intensity by tracking the entire course of microbubble transit through a microvessel, with a 3-fold increase in femoral artery vasa vasorum microvascular density after blood injection compared to the saline-exposed group (201 \pm 11 vessels versus $76 \pm 10$ vessels per section, $P<0.05$ ). Therefore, CEUS with MIP represents an advantage over conventional methods by providing higher average intensities with data less affected by microbubble concentration. The presence of microvessel in the femoral artery adventitia could be detected at 2 weeks using CEUS, indicating the improved accuracy of CEUS with MIP processing for evaluation of vasa vasorum functional density.

In summary, CEUS represents a new opportunity for noninvasive in vivo imaging of atherosclerotic plaques, and its clinical value has been confirmed in both animal experiments and human studies to be a feasible imaging method for visualization of adventitial vasa vasorum neovascularization. As shown in the above-mentioned studies, there is a positive correlation between vasa vasorum density and atherosclerosis and plaque progression. Furthermore, CEUS demonstrates that vasa vasorum was inhibited due to use of antiatherosclerotic drugs, with the development of atherosclerosis being delayed [42]. However, differentiation of stable from unstable plaques by CEUS could be difficult as shown in a recent study by Vavuranakis et al. [43]. CEUS showed the increase in brightness in carotid plaques which correlated with plaque neovessels for stable plaques, but this was not observed in unstable plaques, although more contrast 


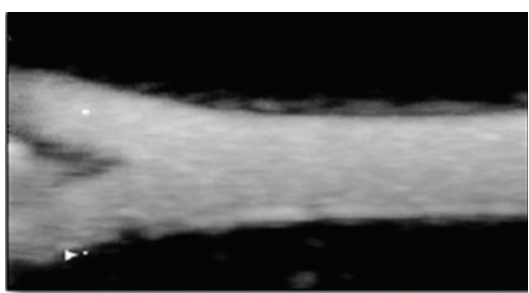

(a)

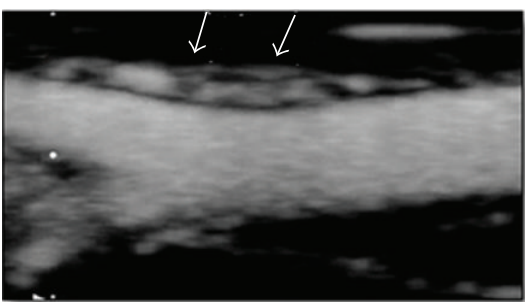

(b)

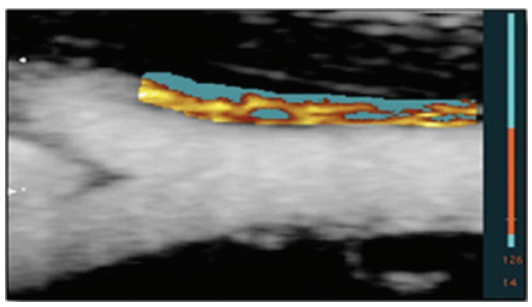

(c)

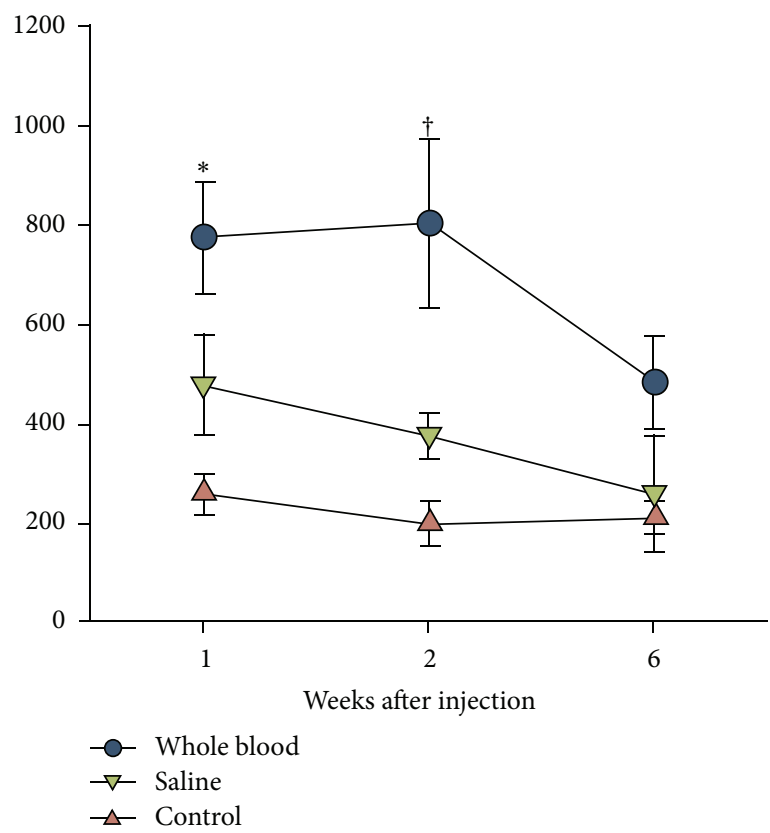

(d)

FIGURE 2: Vasa vasorum blood volume on contrast-enhanced ultrasound imaging. Examples of maximum intensity projection images $2 \mathrm{~s}$ after the destructive pulse sequence are shown for femoral arteries 2 weeks after injection of either saline (a) or whole blood (b), illustrating a greater vasa vasorum (VV) density (arrows) in the latter. (c) Example of pixel intensity threshold analysis for the blood-injected vessel whereby pixels within the region of interest that enhance beyond threshold intensity are displayed in red-orange color scale and those that do not are displayed in blue. (d) Mean ( \pm standard error of the mean) area of enhancement on pixel intensity threshold analysis, an index of functional VV blood density. Data for contralateral noninjected control vessels were similar between treatment cohorts and are grouped. ${ }^{*} P<0.05$ versus control contralateral artery; ${ }^{\dagger} P<0.05$ versus both contralateral and saline-injected arteries (corrected for multiple comparisons). Reprint with permission from [41].

enhancement would be expected to be present in unstable plaque reflecting a more pronounced vascularization. Therefore, further studies are needed to verify the presence of vasa vasorum in atherosclerotic plaques, particularly focusing on the unstable or vulnerable plaques.

2.3. Intravascular Ultrasound. Intravascular ultrasound (IVUS) provides high-resolution tomographic images of the lumen and it is a widely used method for assessing atherosclerotic coronary lesions, guiding stent deployments $[44,45]$. IVUS can be used to acquire precise and reproducible measurements of atherosclerotic plaques in vivo and serially assess the effects of pharmacological treatment on plaque over time in human and animal studies [46]. IVUS imaging systems which are developed to examine flow within the lumen of large arteries are not able to detect vasa vasorum [47]. Using contrast agents has been reported to induce IVUS echogenicity enhancement in the adventitia of coronary arteries, consistent with the detection of vasa vasorum [48]. Studies on animal experiments and human subjects demonstrated the potential of evaluating vasa vasorum in atherosclerotic plaques using IVUS.

Goertz et al. demonstrated the feasibility of using contrast-enhanced IVUS for visualization of vasa vasorum in phantom and vivo experiments $[49,50]$. They used the harmonic contrast imaging method to detect microvascular flow so as to assess the small adventitial vessels consistent with the detection of vasa vasorum. Moritz et al. in a porcine model showed that IVUS allowed for assessment of the density of vasa vasorum [51]. Quantification of the total vasa vasorum flow was performed in their study by summing the blood flow within the vasa vasorum using IVUS, and this was comparable to the quantification of the $3 \mathrm{D}$ distribution of vasa vasorum assessed by micro-CT. Their results demonstrated a high and significant correlation between IVUS and micro-CT in vivo in the visualization of vasa vasorum, which is an indicator of atherosclerotic changes.

Clinical studies on patients further confirmed these experimental results. O’Malley et al. used microbubble contrast-enhanced IVUS in 7 patients with coronary artery 
disease to image and analyse the density and perfusion of vasa vasorum in atherosclerotic plaques [52]. Their results, for the first time, represented the in vivo imaging of vasa vasorum by IVUS. Vavuranakis et al. in their clinical study consisting of 16 patients with acute coronary syndrome validated the feasibility of detecting microbubbles in the coronary lumen using contrast-enhanced IVUS (Figure 3) [53]. The authors reported that contrast-enhanced IVUS enabled quantitative analysis of the echogenicity of the intima-media, adventitia and combined intima-media and adventitia with significant enhancement observed following injection of contrast medium (intima-media: from $6.0 \pm 2.5$ to $7.9 \pm 3.3 \mathrm{~mm}$, $P=0.006$ and adventitia: from $7.1 \pm 2.2$ to $7.6 \pm 2.5 \mathrm{~mm}$, $P=0.035)$. These changes are consistent with vasa vasorum microvessels, which are characteristic features of vulnerable plaques.

In summary, IVUS is a promising technique for detecting the increased volume of blood flow in the vasa vasorum, which forms a basis for analysing atherosclerotic plaques. Although IVUS provides important information about changes in the material content in the arterial wall, it has limited value in quantifying the density of vasa vasorum in the arterial wall. Combined CEUS and IVUS may enhance the diagnostic value of IVUS by providing an opportunity to obtain more direct information in the vascular biology, as shown in a recent study [42].

2.4. Optical Coherence Tomography. Optical coherence tomography (OCT) is a recently developed intravascular imaging modality using near-infrared light to generate cross-sectional intravascular images [54-57]. The imaging principle of OCT is that the electric field amplitude of light reflected from the sample at a certain depth is measured using the principle of low coherence interferometry, with a short coherence length of the source of radiation [58, 59]. The intensity of the interferometric signal is converted to a color-scale or gray-scale to produce cross-sectional images of tissue sample. There are two types of OCT systems that are available to provide intravascular images: first generation OCT systems, known as Time Domain (TD) OCT [58, 59], and second generation systems, known as Fourier Domain (FD) $[60,61]$. TD-OCT uses a broadband light source in the range between 1280 and $1350 \mathrm{~nm}$ band to perform multiple scanning of reference delay distance and directly measure the electric field amplitude. By contrast, FD-OCT uses a monochromatic laser with wavelength changing over time, while the reference delay distance remains constant, and the electric field amplitude is computed through Fourier transformation and is detected at all depth points simultaneously.

The greatest advantage of OCT is its high resolution (10$20 \mu \mathrm{m}$ ), which is 10 times higher than that of IVUS and is comparable to that of micro-CT. OCT can differentiate three layers of the artery wall by showing the adventitia as signal rich layer surrounding the signal poor layer of the media and signal rich layer of the intima closest to the lumen [62, 63]. OCT also allows tissue characterization by identifying three types of plaques, such as fibrous, fibrocalcific, and lipid; therefore, OCT is regarded as a suitable imaging modality for quantifying the thickness of thin cap fibroatheroma and estimating macrophage distribution (vulnerable plaques) [64$66]$. OCT has the ability to characterize these microscopic features of vulnerable plaques which makes it a unique imaging modality.

Following the successful first application of OCT in 10 patients for assessment of plaque vulnerability [67], a number of clinical studies have been performed to evaluate the association between vasa vasorum and atherosclerotic plaques, in particular, the vulnerable plaques using OCT technique. Yabushita et al. in their vitro study examined 357 diseased atherosclerotic arterial segments with results compared to histologic examination [68]. OCT was found to have sensitivity and specificity of $71 \%$ to $79 \%$ and $97 \%$ to $98 \%$ for fibrous plaques, $95 \%$ to $96 \%$ and $97 \%$ for fibrocalcific plaques, and $90 \%$ to $94.5 \%$ and $90 \%$ to $92 \%$ for lipid-rich plaques, respectively. Kitabata et al. showed that microchannels were detected in $38 \%$ of culprit plaques using OCT, with a significant difference observed in the frequency of thin cap fibroatheroma between patients with and without microchannels (54\% versus $21 \%, P=0.012$ ) (Figure 4) [69]. In addition, the thickness of the fibrous cap was significantly thinner in the patients with microchannels $(60 \mu \mathrm{m}$ versus $100 \mu \mathrm{m}, P=0.001)$. Other studies further confirmed the potential value of OCT in evaluating vulnerable plaques in patients with coronary artery disease based on short-to longterm follow-up.

Uemura et al. used OCT to analyse 53 patients with coronary artery disease consisting of 69 nonsignificant coronary stenosis plaques (NSCPs) in terms of plaque characteristics and plaque progression at a mean follow-up of 7 months [70]. Their results showed a significantly higher incidence of intimal laceration (61.5\% versus $8.9 \%$ ), microchannels ( $76.9 \%$ versus $14.3 \%)$, lipid pools (100\% versus $60.7 \%)$, thin cap fibroatheroma (76.9\% versus $14.3 \%)$, macrophage image (61.6\% versus $14.3 \%$ ), and intraluminal thrombus (30.8\% versus $1.8 \%$ ) in NSCPs with progression than those with NSCPs without progression ( $P<0.05$ for all of these comparisons). Barlis et al. showed that OCT could be safely used in vivo to show the culprit coronary lesions and detect morphologic features associated with plaque vulnerability [71]. During 24 months of follow-up of 23 patients prior to coronary angioplasty, OCT detected 7 thin cap fibroatheroma lesions in 6 patients with a mean cap thickness of $0.19 \pm 0.05 \mathrm{~mm}$. Their results together with others indicated that OCT is a valuable technique for assessing culprit atherosclerotic lesions in vivo with favourable results achieved $[72,73]$.

One important aspect about vasa vasorum is that they are dynamic, and the blood flow in these structures can be detected by OCT technique. Cheng and colleagues developed an intensity kurtosis OCT technique to visualize vasa vasorum of carotid artery in vivo [74]. The filling of blood into the vasa vasorum and dynamic motions of the arterial wall were clearly demonstrated using their OCT technique. Their method may provide useful information for evaluating the health status of the artery through imaging of vasa vasorum and associated abnormal changes such as proliferation, thus, 


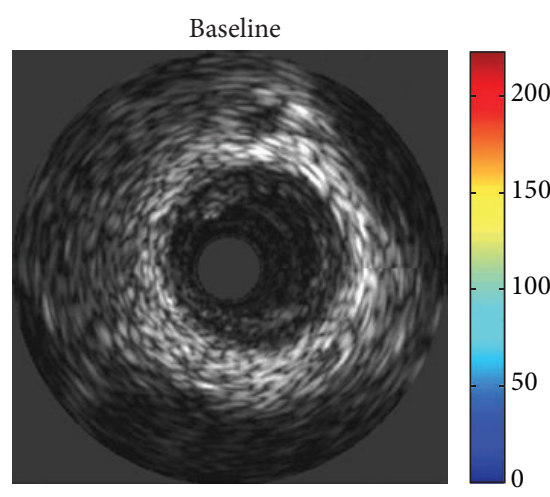

(a)

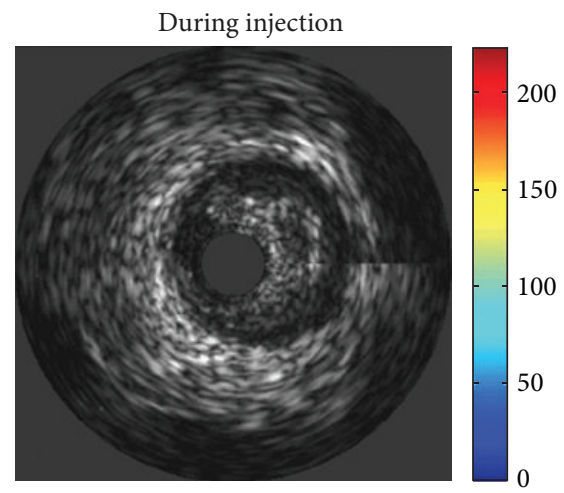

(b)

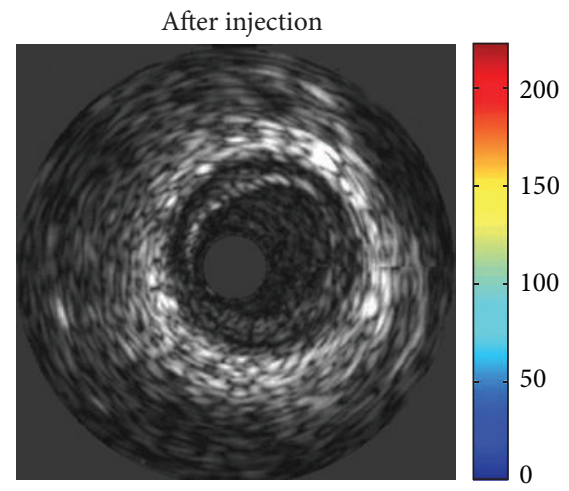

(c)

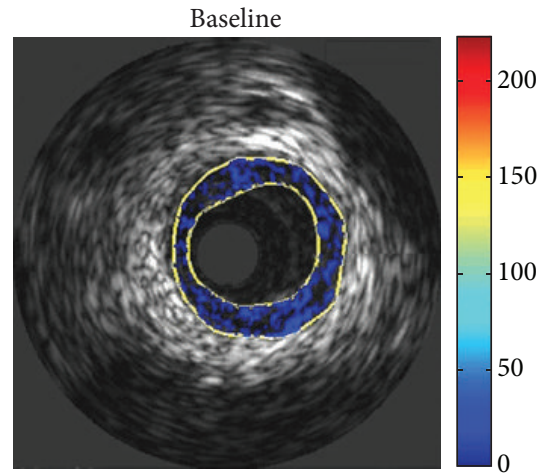

(d)

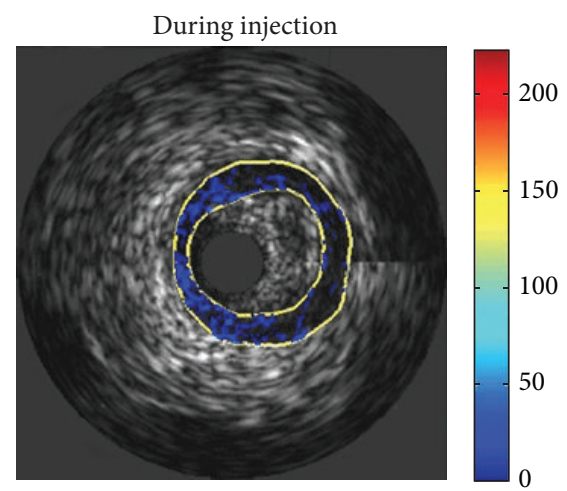

(e)

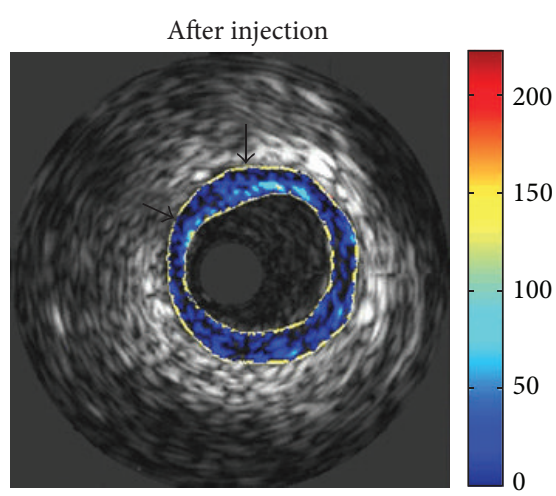

(f)

FIGURE 3: Depiction of qualitative representation of enhancement. Unprocessed images are displayed (a) before, (b) during, and (c) after injection of microbubbles. Corresponding processed images are displayed in (d)-(f). Enhancement is graded from minimal (blue) to maximal (red). Values are a percentage of the maximum grey level intensity difference (255). Arrows indicate points of intense, stable enhancement at the media-adventitia border. Diffuse points of enhancement are present nearby. Reprint with permission from [53].

improving treatment of atherosclerosis in coronary and carotid arteries, although further research is required to verify their early results.

In summary, OCT is an exciting light-based imaging modality with excellent spatial resolution and a strong contrast between the lumen and artery wall structure $[75,76]$. OCT has been validated in both in vitro and in vivo studies to characterize plaque components and identify microvessels and vulnerable plaques which are seen with thin fibrous cap and increased neovascularization of atherosclerosis [70-73, 77-79]. OCT suffers from some limitations. It is not widely available in many catheterization centres. Another main limitation of OCT is the limited depth penetration through tissues, which is less than $2 \mathrm{~mm}$. This will significantly affect the role of OCT to assess plaque burden [56]. Quantitative analysis of subtle morphological parameters such as fibrous cap thickness or compositional parameters could be challenging and may need trained reader interpretation [80]. Furthermore, OCT has been reported to have a moderate diagnostic value in identifying hemodynamically significant coronary stenosis when compared with fractional flow reserve and IVUS [81]. Recently, international guidelines 


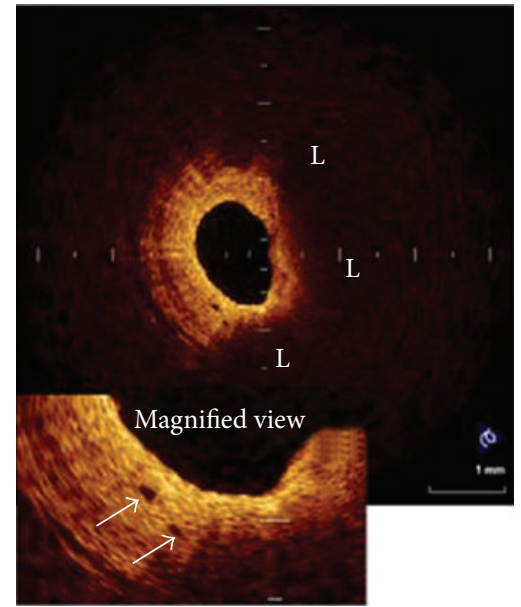

(a)

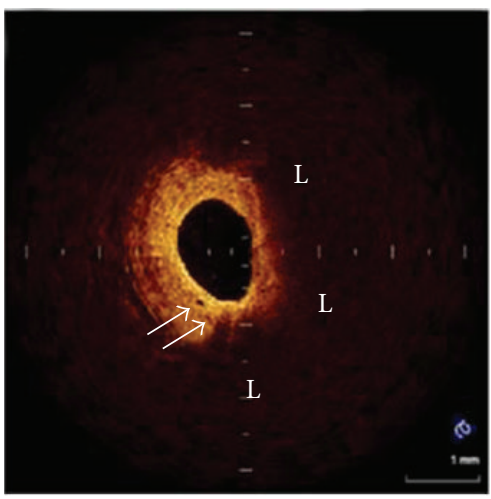

(b)

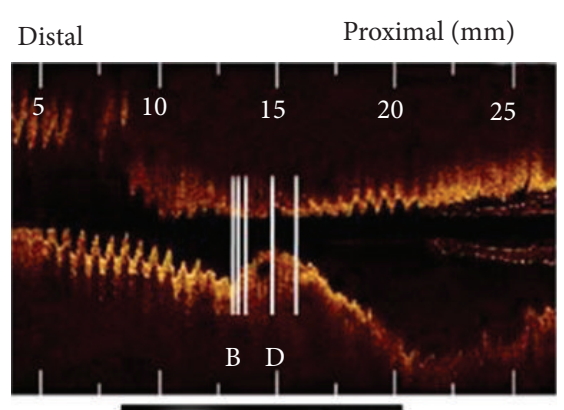

Lesion segment

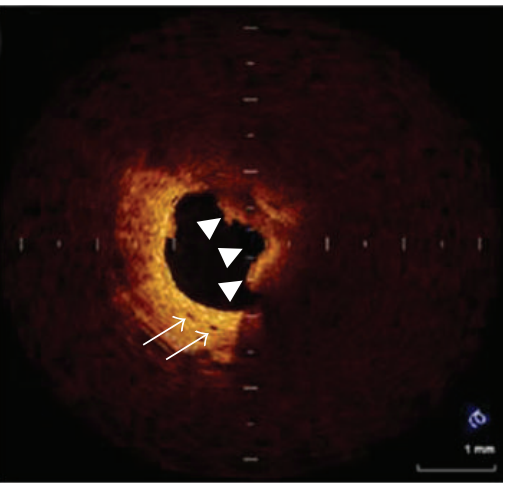

(c)

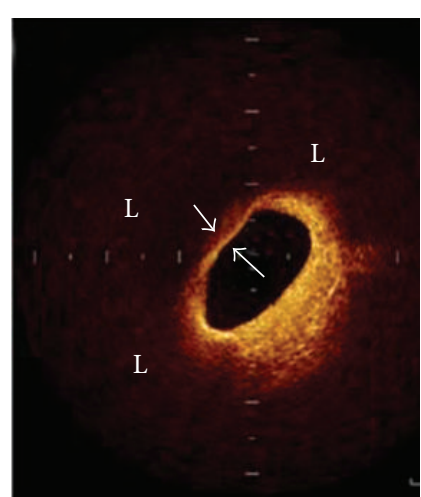

(e)

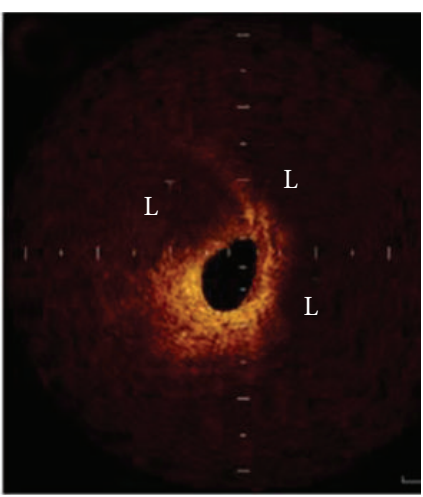

(d)

FIGURE 4: Representative OCT images of coronary plaque with microchannels. (a), (b) Two consecutive cross-sectional OCT images. Eccentric lipid-rich plaque (L) was imaged. Two microchannels (arrow) were located in thickened intima at shoulder region of plaque. (c) Proximal site adjacent to plaque imaged in Figures (a) and (b). Two microchannels (arrow) were located in thickened intima at 7-oclock position. Intracoronary thrombus (arrowhead) was also visualized. (d) Minimum lumen area site. Lipid-rich plaque was visualized. (e) Lipidrich plaque covered by thin fibrous cap $(50 \mu \mathrm{m})$ imaged and found to be thin cap fibroatheroma. Reprint with permission from [69].

have been developed by the International Working Group for Intravascular OCT Standardization and Validation (IWGIVOCT) [82]. The document is recommended for being broadly used as a standard reference regarding the current state of the IVOCT imaging modality in clinical practice.

\subsection{Contrast-Enhanced MRI. MRI is currently recognized} as one of the most valuable imaging modalities for the quantification of vascular plaque burden and assessment of atherosclerotic plaque composition [83-85]. It is well understood that neovascularization of the vessel wall plays a key role in atherosclerotic plaque development and progression; thus, increased neovascularization is often associated with markers of plaque vulnerability such as intraplaque hemorrhage and thin cap fibroatheroma $[86,87]$. Neovessels arising from the vasa vasorum provide nutrients to thickening walls and serve as an entry site for inflammatory cells into the plaque $[88,89]$. Thus, the extent of vasa vasorum may be associated with atherosclerotic plaque size, vascularity, composition, and inflammation [90].
Several different MRI techniques have been developed to assess plaque neovascularization in recent years, and dynamic contrast-enhanced MRI (DCE-MRI) represents a promising technique as it allows for the assessment of neovascular architecture and functional characteristics such as fractional plasma volume and permeability [92, 93]. DCE-MRI also offers high spatial resolution that enables localization of the adventitial boundary, which is the source of the vasa vasorum $[94,95]$.

Cornily et al. in their animal experiments on abdominal aorta acquired from 10 rabbits ( 7 atherosclerotic and 3 control rabbits) showed a significant enhancement in the atherosclerotic group following injection of MRI contrast agent [90]. A strong correlation was found between plaque enhancement and plaque neovessel density using contrastenhanced MRI. Kerwin et al. used DCE-MRI to perform quantitative analysis of the adventitia in patients with carotid artery disease [95]. In 25 patients with carotid endarterectomy specimens, DCE-MRI showed a significant correlation between adventitial transfer constant with the amount of 


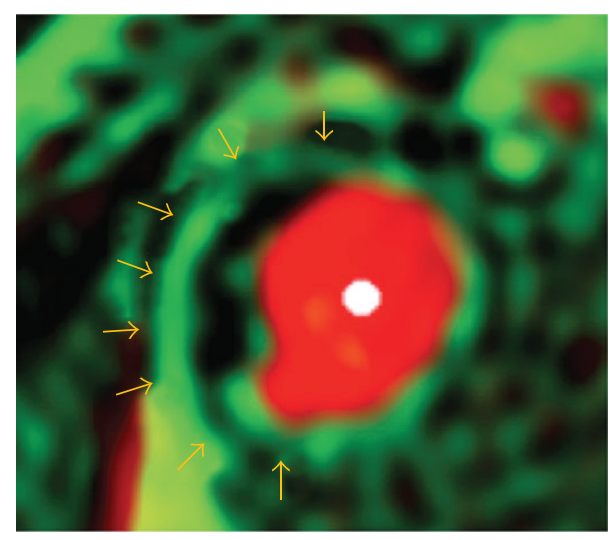

Baseline

(a)

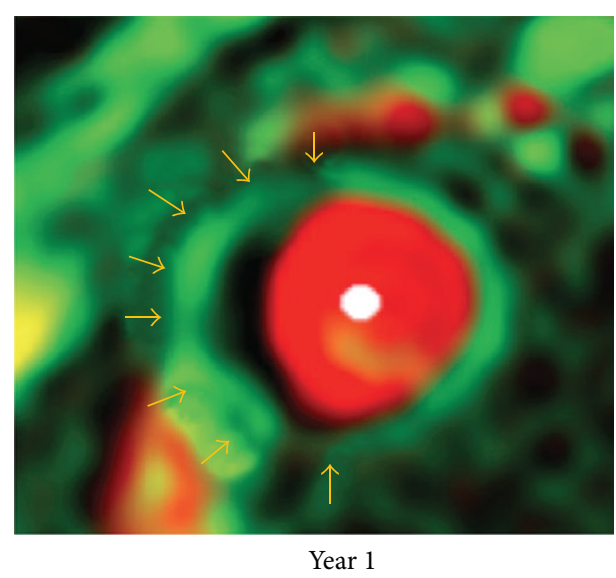

(b)

FIGURE 5: Vasa vasorum images in a 63-year-old man with hypertension. Adventitial transfer constant was 0.129 min $^{-1}$ before therapy and $0.099 \mathrm{~min}^{-1}$ after 1-year treatment. There was subtle gradation of color in adventitial zone (arrows). Reprint with permission from [91].

neovasculature and macrophage $(P<0.05)$. The average transfer constant was significantly higher in patients with severe carotid stenosis than that in patients with moderate disease $\left(0.155 \pm 0.045 \mathrm{~min}^{-1}\right.$ versus $0.122 \pm 0.029 \mathrm{~min}^{-1}, P<$ $0.01)$. The transfer of the contrast agent to the adventitia depends on the vasa vasorum; therefore, adventitial transfer constant is regarded as an indicator to reflect the extent of the vasa vasorum [91, 95]. This is further validated by a recent study by Dong et al. who studied 28 patients using DCE-MRI at both baseline and 1-year follow-up [91]. After 12 months of lipid therapy, a significant reduction was observed in mean adventitial transfer constant when compared to the baseline $\left(0.067 \mathrm{~min}^{-1} \pm 0.028\right.$ versus $0.085 \mathrm{~min}^{-1} \pm 0.037$, $P=0.02$ ) (Figure 5). The adventitial transfer constant was reduced due to the reduction of volume, permeability, or both in neovessels arising from the adventitia. These findings suggest that DCE-MRI could be used as an effective method for the assessment of therapeutic effects on carotid artery atherosclerosis.

In summary, studies have confirmed that contrastenhanced MRI, in particular, DCE-MRI, provides unique advantages to visualize components of atherosclerotic plaques and identify vulnerable plaques in vivo imaging [96-101]. There is a good correlation between atherosclerotic plaque enhancement on MRI imaging and neovessel density of the plaques and signs of inflammation in the plaques in patients with acute coronary syndrome [102-104]. Thus, contrast-enhanced MRI is considered to be a reliable noninvasive tool to detect and characterize features of vulnerable plaques in vivo.

\section{Summary and Conclusion}

Rapid technological advances in imaging modalities have augmented the ability of these imaging techniques to detect and characterize cardiovascular disease, offering information beyond the traditional assessment of lumen changes. Micro-CT demonstrates excellent anatomical details of vasa vasorum due to its superior resolution; however, it is only limited to animal or human cadaver experiments because of its limited field of view which is an inherent disadvantage of the micro-CT scanner. IVUS and OCT are an intravascular imaging modality with unique characteristics of providing superior information on the vascular lumen, plaque components, and vasa vasorum. In particular, OCT has superior resolution which enables it to provide detailed structural information such as different types of plaques. However, both imaging techniques are invasive and are restricted to limited clinical centres. Furthermore, methodology of image acquisition and interpretation needs to be standardized.

CEUS and DCE-MRI both provide important insight into plaque components and microvascular features which are associated with plaque vulnerability; thus, these two noninvasive imaging modalities serve as an effective means for assessing risk status of atherosclerotic plaques and monitoring therapeutic outcomes of atherosclerotic vascular disease. Large prospective clinical trials are needed to demonstrate how these modalities improve patient care by modifying patient management and outcome.

\section{Conflict of Interests}

The author declares that there is no conflict of interests regarding the publication of this paper.

\section{References}

[1] R. Ross, "Atherosclerosis-an inflammatory disease," The New England Journal of Medicine, vol. 340, no. 2, pp. 115-126, 1999.

[2] M. Gössl, N. M. Malyar, M. Rosol, P. E. Beighley, and E. L. Ritman, "Impact of coronary vasa vasorum functional structure on coronary vessel wall perfusion distribution," American Journal of Physiology, vol. 285, no. 5, pp. H2019-H2026, 2003.

[3] M. Gössl, M. Rosol, N. M. Malyar et al., "Functional anatomy and hemodynamic characteristics of vasa vasorum in the walls 
of porcine coronary arteries," Anatomical Record A, vol. 272, no. 2, pp. 526-537, 2003.

[4] M. Gössl, M. Zamir, and E. L. Ritman, "Vasa vasorum growth in the coronary arteries of newborn pigs," Anatomy and Embryology, vol. 208, no. 5, pp. 351-357, 2004.

[5] J. F. Granada and S. B. Feinstein, "Imaging of the vasa vasorum," Nature Clinical Practice Cardiovascular Medicine, vol. 5, no. 2, pp. S18-S25, 2008.

[6] A. C. Barger, R. Beeuwkes 3rd, L. L. Lainey, and K. J. Silverman, "Hypothesis: vasa vasorum and neovascularization of human coronary arteries. A possible role in the pathophysiology of atherosclerosis," The New England Journal of Medicine, vol. 310, no. 3, pp. 175-177, 1984.

[7] M. Kumamoto, Y. Nakashima, and K. Sueishi, "Intimal neovascularization in human coronary atherosclerosis: its origin and pathophysiological significance," Human Pathology, vol. 26, no. 4, pp. 450-456, 1995.

[8] Y. Zhang, W. J. Cliff, G. I. Schoefl, and G. Higgins, "Immunohistochemical study of intimal microvessels in coronary atherosclerosis," American Journal of Pathology, vol. 143, no. 1, pp. 164-172, 1993.

[9] H. A. Hildebrandt, M. Gossl, D. Mannheim et al., "Differential distribution of vasa vasorum in different vascular beds in humans," Atherosclerosis, vol. 199, no. 1, pp. 47-54, 2008.

[10] O. Galili, J. Herrmann, J. Woodrum, K. J. Sattler, L. O. Lerman, and A. Lerman, "Adventitial vasa vasorum heterogeneity among different vascular beds," Journal of Vascular Surgery, vol. 40, no. 3, pp. 529-535, 2004.

[11] S. M. Jorgensen, O. Demirkaya, and E. L. Ritman, "Threedimensional imaging of vasculature and parenchyma in intact rodent organs with X-ray micro-CT," American Journal of Physiology, vol. 275, no. 3, pp. H1103-H1114, 1998.

[12] R. Moritz, D. R. Eaker, A. C. Langheinrich, S. M. Jorgensen, R. M. Bohle, and E. L. Ritman, "Quantification of vasa vasorum density in multi-slice computed tomographic coronary angiograms: role of computed tomographic image voxel size," Journal of Computer Assisted Tomography, vol. 34, no. 2, pp. 273278, 2010.

[13] Z. Sun, G. H. Choo, and K. H. Ng, "Coronary CT angiography: current status and continuing challenges," British Journal of Radiology, vol. 85, pp. 495-510, 2012.

[14] H. M. Kwon, G. Sangiorgi, E. L. Ritman et al., "Enhanced coronary vasa vasorum neovascularization in experimental hypercholesterolemia," Journal of Clinical Investigation, vol. 101, no. 8, pp. 1551-1556, 1998.

[15] J. Herrmann, L. O. Lerman, M. Rodriguez-Porcel et al., "Coronary vasa vasorum neovascularization precedes epicardial endothelial dysfunction in experimental hypercholesterolemia," Cardiovascular Research, vol. 51, no. 4, pp. 762-766, 2001.

[16] J. Herrmann, P. J. Best, E. L. Ritman, D. R. Holmes Jr., L. O. Lerman, and A. Lerman, "Chronic endothelin receptor antagonism prevents. Coronary vasa vasorum neovascularization in experimental hypercholesterolemia," Journal of the American College of Cardiology, vol. 39, no. 9, pp. 1555-1561, 2002.

[17] M. Gössl, J. Herrmann, H. Tang et al., "Prevention of vasa vasorum neovascularization attenuates early neointima formation in experimental hypercholesterolemia," Basic Research in Cardiology, vol. 104, no. 6, pp. 695-706, 2009.
[18] M. Gössl, D. Versari, D. Mannheim, E. L. Ritman, L. O. Lerman, and A. Lerman, "Increased spatial vasa vasorum density in the proximal LAD in hypercholesterolemia-implications for vulnerable plaque-development," Atherosclerosis, vol. 192, no. 2, pp. 246-252, 2007.

[19] S. H. Wilson, J. Herrmann, L. O. Lerman et al., "Simvastatin preserves the structure of coronary adventitial vasa vasorum in experimental hypercholesterolemia independent of lipid lowering," Circulation, vol. 105, no. 4, pp. 415-418, 2002.

[20] P. R. Hansen, J. H. Svendsen, S. Hoyer, A. Kharazmi, K. Bendtzen, and S. Haunso, "Tumor necrosis factor- $\alpha$ increases myocardial microvascular transport in vivo," American Journal of Physiology, vol. 266, no. 1, pp. H60-H67, 1994.

[21] J. M. Isner, "Vasa vasorum: therapeutic implications," Catheterization and Cardiovascular Diagnosis, vol. 39, no. 3, pp. 221-223, 1996.

[22] B. M. Fu and S. Shen, "Acute VEGF effect on solute permeability of mammalian microvessels in vivo," Microvascular Research, vol. 68, no. 1, pp. 51-62, 2004.

[23] M. Gössl, D. Versari, H. A. Hildebrandt et al., "Segmental heterogeneity of vasa vasorum neovascularization in human coronary atherosclerosis," Journal of the American College of Cardiology, vol. 3, no. 1, pp. 32-40, 2010.

[24] Z.-H. Sun, Y. Cao, and H.-F. Li, "Multislice computed tomography angiography in the diagnosis of coronary artery disease," Journal of Geriatric Cardiology, vol. 8, no. 2, pp. 104-113, 2011.

[25] K. Kajinami, H. Seki, N. Takekoshi, and H. Mabuchi, "Coronary calcification and coronary atherosclerosis: site by site comparative morphologic study of electron beam computed tomography and coronary angiography," Journal of the American College of Cardiology, vol. 29, no. 7, pp. 1549-1556, 1997.

[26] M. Gössl, U. I. Mödder, E. J. Atkinson, A. Lerman, and S. Khosla, "Osteocalcin expression by circulating endothelial progenitor cells in patients with coronary atherosclerosis," Journal of the American College of Cardiology, vol. 52, no. 16, pp. 1314-1325, 2008.

[27] M. Kampschulte, A. Brinkmann, P. Stieger et al., "Quantitative CT imaging of the spatio-temporal distribution patterns of vasa vasorum in aortas of apoE ${ }^{-} /^{-} / \mathrm{LDL}^{-} /^{-}$double knockout mice," Atherosclerosis, vol. 212, no. 2, pp. 444-450, 2010.

[28] R. Moritz, J. L. Anderson, A. J. Vercnocke, R. J. Wentz, and E. L. Ritman, "Changes in CT angiographic opacification of porcine coronary artery wall with patchy altered flow in vasa vasorum," International Journal of Cardiovascular Imaging, vol. 29, pp. 1325-1333, 2013.

[29] S. B. Feinstein, "Contrast ultrasound imaging of the carotid artery vasa vasorum and atherosclerotic plaque neovascularization," Journal of the American College of Cardiology, vol. 48, no. 2, pp. 236-243, 2006.

[30] S. B. Feinstein, "The powerful microbubble: from bench to bedside, from intravascular indicator to therapeutic delivery system, and beyond," American Journal of Physiology, vol. 287, no. 2, pp. H450-H457, 2004.

[31] D. Staub, A. F. L. Schinkel, B. Coll et al., "Contrast-enhanced ultrasound imaging of the vasa vasorum: from early atherosclerosis to the identification of unstable plaques," Journal of the American College of Cardiology, vol. 3, no. 7, pp. 761-771, 2010.

[32] D. A. Clevert, W. H. Sommer, A. Helck, T. Saam, and M. Reiser, "Improved carotid atherosclerotic plaques imaging with contrast-enhanced ultrasound (CEUS)," Clinical Hemorheology and Microcirculation, vol. 48, no. 1-3, pp. 141-148, 2011. 
[33] S. Coli, M. Magnoni, G. Sangiorgi et al., "Contrast-enhanced ultrasound imaging of intraplaque neovascularization in carotid arteries. Correlation with histology and plaque echogenicity," Journal of the American College of Cardiology, vol. 52, no. 3, pp. 223-230, 2008.

[34] A. Hoogi, D. Adam, A. Hoffman, H. Kerner, S. Reisner, and D. Gaitini, "Carotid plaque vulnerability: quantification of neovascularization on contrast-enhanced ultrasound with histopathologic correlation," American Journal of Roentgenology, vol. 196, no. 2, pp. 431-436, 2011.

[35] C. Giannarelli, B. Ibanez, G. Cimmino et al., "Contrastenhanced ultrasound imaging detects intraplaque neovascularization in an experimental model of atherosclerosis," Journal of the American College of Cardiology, vol. 3, no. 12, pp. 1256-1264, 2010.

[36] A. F. L. Schinkel, C. G. Krueger, A. Tellez et al., "Contrastenhanced ultrasound for imaging vasa vasorum: comparison with histopathology in a swine model of atherosclerosis," European Journal of Echocardiography, vol. 11, no. 8, pp. 659$664,2010$.

[37] M. F. Giannoni, E. Vicenzini, M. Citone et al., "Contrast carotid ultrasound for the detection of unstable plaques with neoangiogenesis: A Pilot Study," European Journal of Vascular and Endovascular Surgery, vol. 37, no. 6, pp. 722-727, 2009.

[38] L. Xiong, Y.-B. Deng, Y. Zhu, Y.-N. Liu, and X.-J. Bi, "Correlation of carotid plaque neovascularization detected by using contrast-enhanced US with clinical symptoms," Radiology, vol. 251, no. 2, pp. 583-589, 2009.

[39] F. Shah, P. Balan, M. Weinberg et al., "Contrast-enhanced ultrasound imaging of atherosclerotic carotid plaque neovascularization: a new surrogate marker of atherosclerosis?" Vascular Medicine, vol. 12, no. 4, pp. 291-297, 2007.

[40] E. Vicenzini, M. F. Giannoni, F. Puccinelli et al., "Detection of carotid adventitial vasa vasorum and plaque vascularization with ultrasound cadence contrast pulse sequencing technique and echo-contrast agent," Stroke, vol. 38, no. 10, pp. 2841-2843, 2007.

[41] S. C. Lee, C. L. Carr, B. P. Davidson et al., "Temporal characterization of the functional density of the vasa vasorum by contrast-enhanced ultrasonography maximum intensity projection imaging," Journal of the American College of Cardiology, vol. 3, no. 12, pp. 1265-1272, 2010.

[42] J. Tian, S. Hu, Y. Sun et al., "Vasa vasorum and plaque progression, and responses to atorvastatin in a rabbit model of atherosclerosis: contrast-enhanced ultrasound imaging and intravascular ultrasound study," Heart, vol. 99, pp. 48-54, 2013.

[43] M. Vavuranakis, F. Sigala, D. A. Vrachatis et al., "Quantitative analysis of carotid plaque vasa vasorum by CEUS and correlation with histology after endarterectomy," Vasa, vol. 42, pp. 184195, 2013.

[44] D. Böse, C. von Birgelen, and R. Erbel, "Intravascular ultrasound for the evaluation of therapies targeting coronary atherosclerosis," Journal of the American College of Cardiology, vol. 49, no. 9, pp. 925-932, 2007.

[45] G. S. Mintz, S. E. Nissen, W. D. Anderson et al., "American College of Cardiology clinical expert consensus document on standards for acquisition, measurement and reporting of intravascular ultrasound studies," Journal of the American College of Cardiology, vol. 37, no. 5, pp. 1478-1492, 2001.
[46] I. Sipahi, E. M. Tuzcu, K.-W. Moon et al., "Do the extent and direction of arterial remodelling predict subsequent progression of coronary atherosclerosis? A serial intravascular ultrasound study," Heart, vol. 94, no. 5, pp. 623-627, 2008.

[47] W. Li, A. F. W. Van Der Steen, C. T. Lancée, I. Céspedes, and N. Bom, "Blood flow imaging and volume flow quantitation with intravascular ultrasound," Ultrasound in Medicine and Biology, vol. 24, no. 2, pp. 203-214, 1998.

[48] S. Carlier, A. Kakadiaris, N. Dib et al., "Vasa vasorum imaging: a new window to the clinical detection of vulnerable atherosclerotic plaques," Current Atherosclerosis Reports, vol. 7, pp. 164$169,2005$.

[49] D. E. Goertz, M. E. Frijlink, D. Tempel et al., "Contrast harmonic intravascular ultrasound: a feasibility study for vasa vasorum imaging," Investigative Radiology, vol. 41, no. 8, pp. 631-638, 2006.

[50] D. E. Goertz, M. E. Frijlink, D. Tempel et al., "Subharmonic contrast intravascular ultrasound for vasa vasorum imaging," Ultrasound in Medicine and Biology, vol. 33, no. 12, pp. 18591872, 2007.

[51] R. Moritz, D. R. Eaker, J. L. Anderson et al., "IVUS detection of vas vasorum blood flow distribution in coronary artery vessel wall," Journal of the American College of Cardiology, vol. 5, pp. 935-940, 2012.

[52] S. M. O’Malley, M. Vavuranakis, M. Naghavi, and I. A. Kadadiaris, "Intravascular ultrasound-based imaging of vasa vasorum for the detection of vulnerable atherosclerotic plaque," Medical Imaging Computing and Computer-Assisted Intervention, vol. 8, pp. 343-351, 2005.

[53] M. Vavuranakis, I. A. Kakadiaris, S. M. O’Malley et al., "A new method for assessment of plaque vulnerability based on vasa vasorum imaging, by using contrast-enhanced intravascular ultrasound and differential image analysis," International Journal of Cardiology, vol. 130, no. 1, pp. 23-29, 2008.

[54] T. Akasaka, T. Kubo, M. Mizukoshi et al., "Pathophysiology of acute coronary syndrome assessed by optical coherence tomography," Journal of Cardiology, vol. 56, no. 1, pp. 8-14, 2010.

[55] T. Kubo and T. Akasaka, "Recent advances in intracoronary imaging techniques: focus on optical coherence tomography," Expert Review of Medical Devices, vol. 5, no. 6, pp. 691-697, 2008.

[56] S. Rathore, M. Terashima, H. Matsuo et al., "Association of coronary plaque composition and arterial remodelling: a optical coherence tomography study," Atherosclerosis, vol. 221, no. 2, pp. 405-415, 2012.

[57] G. Ferrante, P. Presbitero, R. Whitbourn, and P. Barlis, "Current applications of optical coherence tomography for coronary intervention," International Journal of Cardiology, vol. 165, pp. 7-16, 2013.

[58] R. C. Youngquist, S. Carr, and D. E. N. Davies, "Optical coherence-domain reflectometry: a new optical evaluation technique," Optics Letters, vol. 12, no. 3, pp. 158-160, 1987.

[59] K. Takada, I. Yokohama, K. Chida, and J. Noda, "New measurement system for fault location in optical waveguide devices based on interferometric technique," Applied Optics, vol. 26, pp. 1603-1606, 1987.

[60] S. H. Yun, G. J. Tearney, J. F. De Boer, N. Iftimia, and B. E. Bouma, "High-speed optical frequency-domain imaging," Optics Express, vol. 11, no. 22, pp. 2953-2963, 2003.

[61] B. E. Bouma, S.-H. Yun, B. J. Vakoc, M. J. Suter, and G. J. Tearney, "Fourier-domain optical coherence tomography: 
recent advances toward clinical utility," Current Opinion in Biotechnology, vol. 20, no. 1, pp. 111-118, 2009.

[62] T. Kume, T. Akasaka, T. Kawamoto et al., "Assessment of coronary intima-media thickness by optical coherence tomography: comparison with intravascular ultrasound," Circulation Journal, vol. 69, no. 8, pp. 903-907, 2005.

[63] T. Kubo, Y. Ino, T. Tanimoto, H. Kitabata, A. Tanaka, and T. Akasaka, "Optical coherence tomography imaging in acute coronary syndromes," Cardiology Research and Practice, vol. 1, no. 1, Article ID 312978, 2011.

[64] B. D. MacNeill, I.-K. Jang, B. E. Bouma et al., "Focal and multifocal plaque macrophage distributions in patients with acute and stable presentations of coronary artery disease," Journal of the American College of Cardiology, vol. 44, no. 5, pp. 972-979, 2004.

[65] O. C. Raffel, G. J. Tearney, D. D. Gauthier, E. F. Halpern, B. E. Bouma, and I.-K. Jang, "Relationship between a systemic inflammatory marker, plaque inflammation, and plaque characteristics determined by intravascular optical coherence tomography," Arteriosclerosis, Thrombosis, and Vascular Biology, vol. 27, no. 8, pp. 1820-1827, 2007.

[66] F. Prati, E. Regar, G. S. Mintz et al., "Expert review document on methodology, terminology, and clinical applications of optical coherence tomography: physical principles, methodology of image acquisition, and clinical application for assessment of coronary arteries and atherosclerosis," European Heart Journal, vol. 31, no. 4, pp. 401-415, 2010.

[67] I.-K. Jang, B. E. Bouma, D.-H. Kang et al., "Visualization of coronary atherosclerotic plaques in patients using optical coherence tomography: comparison with intravascular ultrasound," Journal of the American College of Cardiology, vol. 39, no. 4, pp. 604-609, 2002.

[68] H. Yabushita, B. E. Bouma, S. L. Houser et al., "Characterization of human atherosclerosis by optical coherence tomography," Circulation, vol. 106, no. 13, pp. 1640-1645, 2002.

[69] H. Kitabata, A. Tanaka, T. Kubo et al., "Relation of microchannel structure identified by optical coherence tomography to plaque vulnerability in patients with coronary artery disease," American Journal of Cardiology, vol. 105, no. 12, pp. 1673-1678, 2010.

[70] S. Uemura, K.-I. Ishigami, T. Soeda et al., "Thin-cap fibroatheroma and microchannel findings in optical coherence tomography correlate with subsequent progression of coronary atheromatous plaques," European Heart Journal, vol. 33, no. 1, pp. 78-85, 2012.

[71] P. Barlis, P. W. Serruys, N. Gonzalo, W. J. van der Giessen, P. J. de Jaegere, and E. Regar, "Assessment of culprit and remote coronary narrowings using optical coherence tomography with long-term outcomes," American Journal of Cardiology, vol. 102, no. 4, pp. 391-395, 2008.

[72] I.-K. Jang, G. J. Tearney, B. MacNeill et al., "In vivo characterization of coronary atherosclerotic plaque by use of optical coherence tomography," Circulation, vol. 111, no. 12, pp. 15511555, 2005.

[73] T. Kubo, T. Imanishi, S. Takarada et al., "Assessment of culprit lesion morphology in acute myocardial infarction. Ability of optical coherence tomography compared with intravascular ultrasound and coronary angioscopy," Journal of the American College of Cardiology, vol. 50, no. 10, pp. 933-939, 2007.

[74] K. H. Y. Cheng, C. Sun, B. Vuong et al., "Endovascular optical coherence tomography intensity kurtosis: visualization of vasa vasorum in porcine carotid artery," Biomedical Optical Express, vol. 3, pp. 388-399, 2012.

[75] R. Hamdan, R. G. Gonzalez, S. Ghostine, and C. Caussin, "Optical coherence tomography: from physical principles to clinical applications," Archives of Cardiovascular Disease, vol. 105, pp. 529-534, 2012.

[76] P. P. Karjalainen and W. Nammas, "Optical coherence tomography: on the way to decipher the 'rosetta stone"' International Journal of Cardiology, vol. 159, pp. 79-81, 2012.

[77] R. Puri, E. M. Tuzcu, S. E. Nissen, and S. J. Nicholls, "Exploring coronary atherosclerosis with intravascular imaging," International Journal of Cardiology, vol. 168, no. 2, pp. 670-679, 2013.

[78] A. Tanaka, T. Imanishi, H. Kitabata et al., "Morphology of exertion-triggered plaque rupture in patients with acute coronary syndrome: an optical coherence tomography study," Circulation, vol. 118, no. 23, pp. 2368-2373, 2008.

[79] Y. Ino, T. Kubo, A. Tanaka et al., "Difference of culprit lesion morphologies between ST-segment elevation myocardial infarction and non-ST-segment elevation acute coronary syndrome," Journal of the American College of Cardiology, vol. 4, no. 1, pp. 76-82, 2011.

[80] H. C. Lowe, J. Narula, J. G. Fujimoto, and I.-K. Jang, "Intracoronary optical diagnostics: current status, limitations, and potential," Journal of the American College of Cardiology, vol. 4, no. 12, pp. 1257-1270, 2011.

[81] N. Gonzalo, J. Escaned, F. Alfonso et al., "Morphometric assessment of coronary stenosis relevance with optical coherence tomography: a comparison with fractional flow reserve and intravascular ultrasound," Journal of the American College of Cardiology, vol. 59, pp. 1080-1089, 2012.

[82] G. J. Tearney, E. Regar, T. Akasaka et al., "Consensus standards for acquisition, measurement, and reporting of intravascular optical coherence tomography studies. A report from the International Working Group for Intravascular Optical Coherence Tomography Standardization and Validation," Journal of the American College of Cardiology, vol. 59, pp. 1058-1072, 2012.

[83] C. Yuan, E. Lin, J. Millard, and J.-N. Hwang, "Closed contour edge detection of blood vessel lumen and outer wall boundaries in black-blood MR images," Magnetic Resonance Imaging, vol. 17, no. 2, pp. 257-266, 1999.

[84] Z. A. Fayad, V. Fuster, J. T. Fallon et al., "Noninvasive in vivo human coronary artery lumen and wall imaging using blackblood magnetic resonance imaging," Circulation, vol. 102, no. 5, pp. 506-510, 2000.

[85] R. P. Choudhury, V. Fuster, J. J. Badimon, E. A. Fisher, and Z. A. Fayad, "MRI and characterization of atherosclerotic plaque: emerging applications and molecular imaging," Arteriosclerosis, Thrombosis, and Vascular Biology, vol. 22, no. 7, pp. 1065-1074, 2002.

[86] P. R. Moreno, K. R. Purushothaman, V. Fuster et al., "Plaque neovascularization is increased in ruptured atherosclerotic lesions of human aorta: implications for plaque vulnerability," Circulation, vol. 110, no. 14, pp. 2032-2038, 2004.

[87] R. Virmani, F. D. Kolodgie, A. P. Burke et al., "Atherosclerotic plaque progression and vulnerability to rupture: angiogenesis as a source of intraplaque hemorrhage," Arteriosclerosis, Thrombosis, and Vascular Biology, vol. 25, no. 10, pp. 2054-2061, 2005.

[88] P. R. Moreno, K.-R. Purushothaman, M. Sirol, A. P. Levy, and V. Fuster, "Neovascularization in human atherosclerosis," Circulation, vol. 113, no. 18, pp. 2245-2252, 2006. 
[89] O. J. De Boer, A. C. Van Der Wal, P. Teeling, and A. E. Becker, "Leucocyte recruitment in rupture prone regions of lipid-rich plaques: a prominent role for neovascularization?" Cardiovascular Research, vol. 41, no. 2, pp. 443-449, 1999.

[90] J.-C. Cornily, F. Hyafil, C. Calcagno et al., "Evaluation of neovessels in atherosclerotic plaques of rabbits using an albuminbinding intravascular contrast agent and MRI," Journal of Magnetic Resonance Imaging, vol. 27, no. 6, pp. 1406-1411, 2008.

[91] L. Dong, W. S. Kerwin, H. Chen et al., "Carotid artery atherosclerosis: effect of intensive lipid therapy on the vasa vasorum-evaluation by using dynamic contrast-enhanced MR imaging," Radiology, vol. 260, no. 1, pp. 224-231, 2011.

[92] W. Kerwin, A. Hooker, M. Spilker et al., "Quantitative magnetic resonance imaging analysis of neovasculature volume in carotid atherosclerotic plaque," Circulation, vol. 107, no. 6, pp. 851-856, 2003.

[93] W. S. Kerwin, K. D. O’Brien, M. S. Ferguson, N. Polissar, T. S. Hatsukami, and C. Yuan, "Inflammation in carotid atherosclerotic plaque: a dynamic contrast-enhanced MR imaging study," Radiology, vol. 241, no. 2, pp. 459-468, 2006.

[94] C. Yuan, K. W. Beach, L. H. Smith Jr., and T. S. Hatsukami, "Measurement of atherosclerotic carotid plaque size in vivo using high resolution magnetic resonance imaging," Circulation, vol. 98, no. 24, pp. 2666-2671, 1998.

[95] W. S. Kerwin, M. Oikawa, C. Yuan, G. P. Jarvik, and T. S. Hatsukami, "MR imaging of adventitial vasa vasorum in carotid atherosclerosis," Magnetic Resonance in Medicine, vol. 59, no. 3, pp. 507-514, 2008.

[96] T. Li, X. Zhao, X. Liu et al., "Evaluation of the early enhancement of coronary atherosclerotic plaque by contrast-enhanced MR angiography," European Journal of Radiology, vol. 80, no. 1, pp. 136-142, 2011.

[97] S. Wagner, J. Schnorr, A. Ludwig et al., "Contrast-enhanced MR imaging of atherosclerosis using citrate-coated superparamagnetic iron oxide nanoparticles: calcifying microvesicles as imaging target for plaque characterization," International Journal of Nanomedicine, vol. 8, pp. 767-779, 2013.

[98] B. A. Wasserman, A. R. Sharrett, S. Lai et al., "Risk factor associations with the presence of a lipid core in carotid plaque of asymptomatic individuals using high-resolution MRI: the Multi-Ethnic Study of Atherosclerosis (MESA)," Stroke, vol. 39, no. 2, pp. 329-335, 2008.

[99] L. Wagenknecht, B. Wasserman, L. Chambless et al., "Correlates of carotid plaque presence and composition as measured by mri the atherosclerosis risk in communities study," Circulation, vol. 2, no. 4, pp. 314-322, 2009.

[100] Z. A. Fayad, M. Sirol, P. R. Moreno et al., "Increased neovascularization in advanced lipid-rich atherosclerotic lesions detected by gadofluorine-m-enhanced MRI: implications for plaque vulnerability," Circulation, vol. 2, no. 5, pp. 391-396, 2009.

[101] X. B. Jiang, W. S. Yuan, J. S. Wang et al., "Matrix metalloproteinase-9 expression in carotid atherosclerotic plaque and contrast-enhanced MRI in a swine model," Journal of Neurointerventional Surgery, vol. 6, no. 1, pp. 24-28, 2014.

[102] A. Lombardo, V. Rizzello, L. Natale et al., "Magnetic resonance imaging of carotid plaque inflammation in acute coronary syndromes: a sign of multisite plaque activation," International Journal of Cardiology, vol. 136, no. 1, pp. 103-105, 2009.

[103] T. Saam, J. Cai, L. Ma et al., "Comparison of symptomatic and asymptomatic atherosclerotic carotid plaque features with in vivo MR imaging," Radiology, vol. 240, no. 2, pp. 464-472, 2006.
[104] Y. Qiao, M. Etesami, S. Malhotra et al., "Identification of intraplaque hemorrhage on MR angiography images: a comparison of contrast-enhanced mask and time-of-flight techniques," American Journal of Neuroradiology, vol. 32, no. 3, pp. 454-459, 2011. 


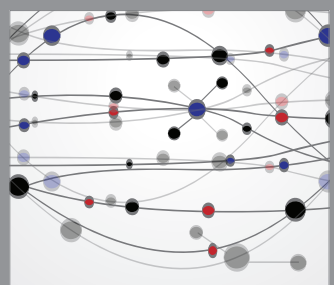

The Scientific World Journal
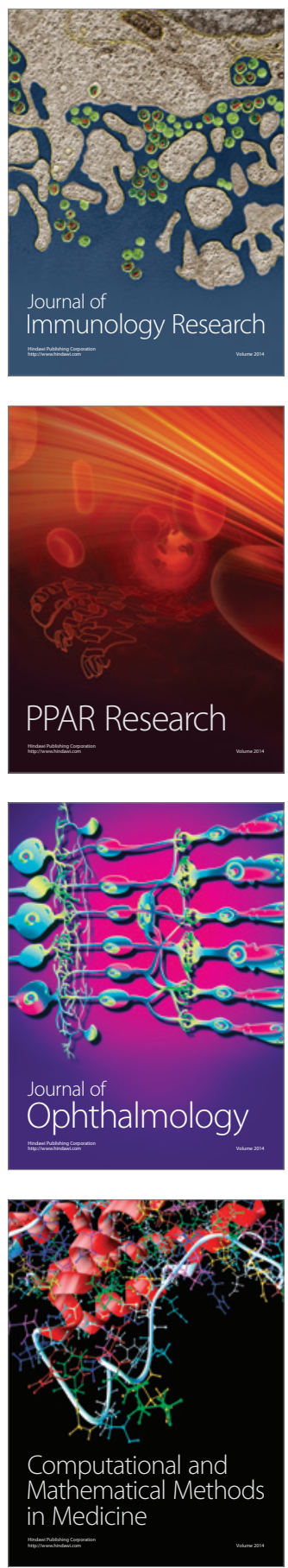

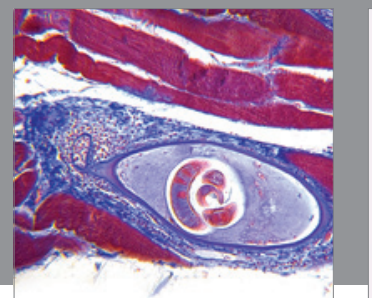

Gastroenterology

Research and Practice
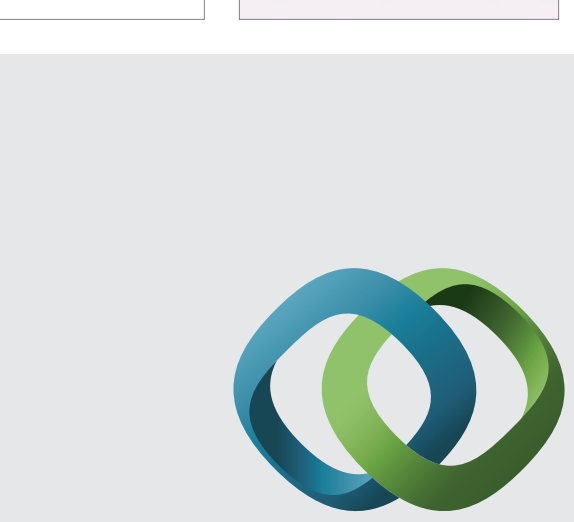

\section{Hindawi}

Submit your manuscripts at

http://www.hindawi.com
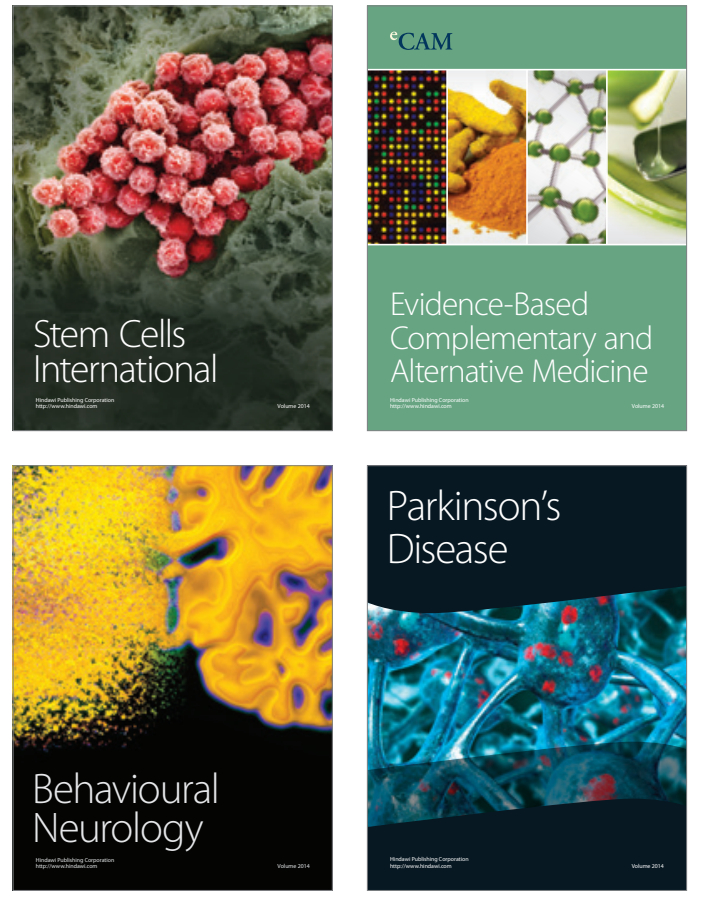
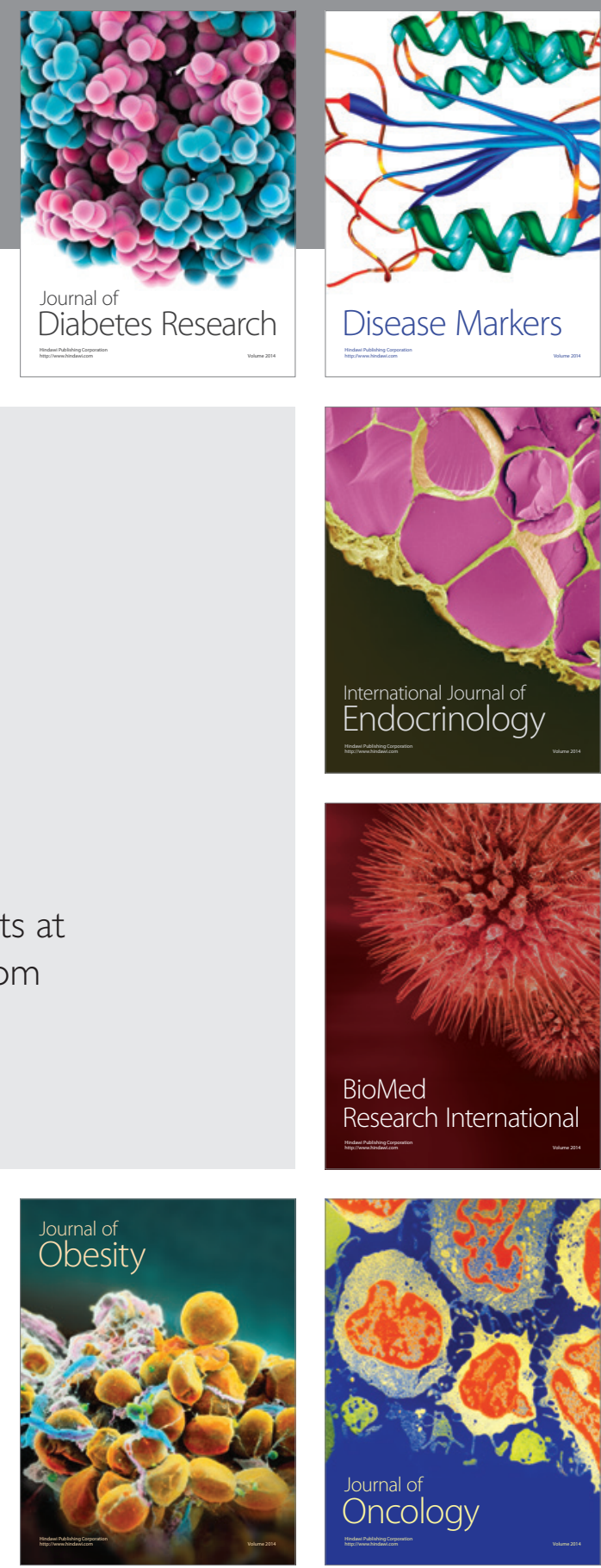

Disease Markers
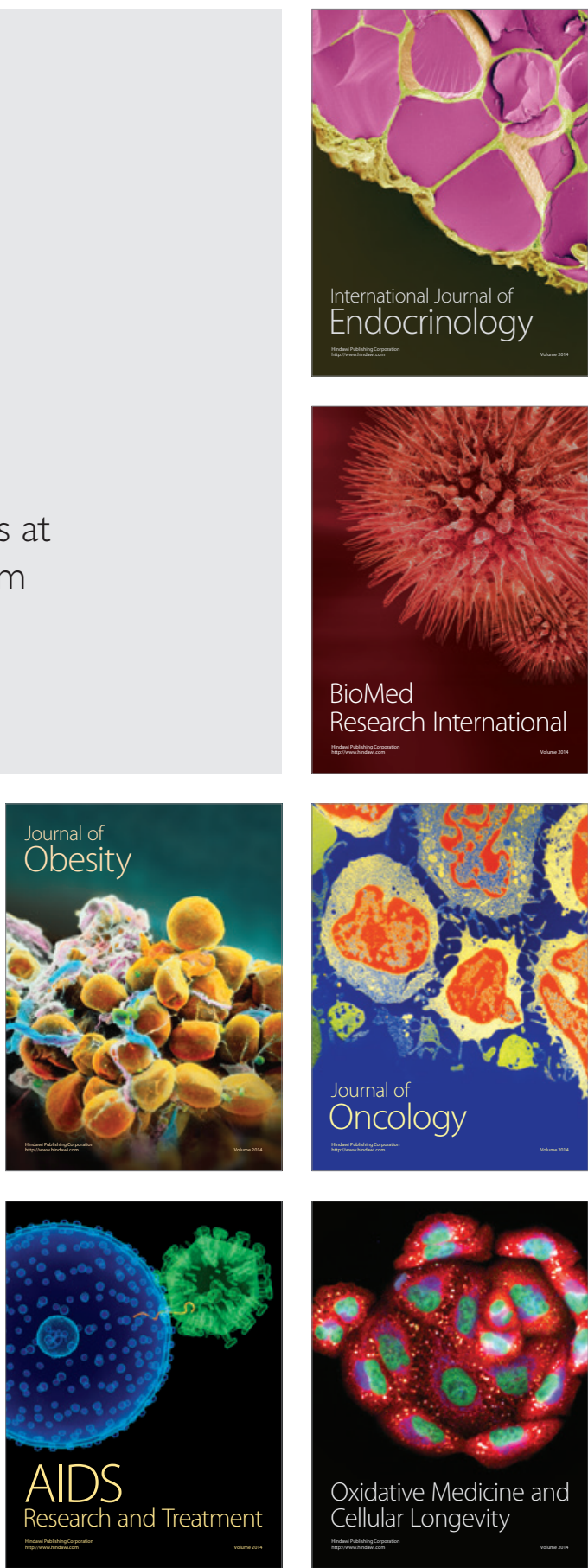